\title{
A View from Down Under
}

I have just returned from the 19th conference of the Society for Ecological Restoration International held in Perth, Western Australia. It was a splendid event: excellent talks and field trips, a fabulous venue, and plenty of opportunity to network, learn, and disseminate good practice and research ideas.

It was heartening to see many young people at the conference, involved in restoration as practitioners, regulators, and academics and freely exchanging experiences and viewpoints. The interplay between research, education, and implementation was what really drew me to SERI in the first instance- the feed forward of ideas from the cutting edge of research melded with indigenous knowledge and the experience of practitioners into new ways of practicing ecological restoration. In turn, those at the cutting edge "on the ground" feed back their experience and problems, initiating new ideas for research, enquiry, and reflection. The conference also gave me an opportunity to reflect on some of the basic principles of restoration that we have developed as a society over the last 20 years.

I was fortunate to take part in a field trip to areas south of Perth to Albany, where we visited areas devastated by saline intrusion caused by rising groundwater tables as a result of vigorous and extensive removal of the native flora. This removal began in the 1800 s and was extended into the 1920s and 1930s. One site had been conserved by heroic groundwater pumping efforts. The interventions needed to restore such systems, catchments that cover very large land areas, require an effort at a national scale. As stated in the Society for Ecological Restoration International Primer, "removing external threats" may involve interaction of stakeholders quite removed from the immediate site of interest. Without this scale of intervention, however, the restoration is destined to fail-or not get going in the first place.
We also saw a restoration project that is part of the large scale restoration initiative Gondwana Link, which aims to reconnect fragmented habitats over large areas of south western Australia. This landscape is a very ancient one; its soils were undisturbed for many hundreds of millennia. The area has developed a stable vegetation type that Stephen Hopper, Director of the Royal Botanic Gardens at Kew calls "OCBIL," old, climatically buffered, infertile landscapes. The flora is dependent on fire and low precipitation and disperses very slowly. The field trip underscored the challenges involved in reinstating the subtle interplay of perturbation and stress that produced this rare vegetation type (along with the South African fynbos and parts of South America that were linked with Gondwana some 200 million years ago). Simply reinstating contours and hydrology isn't enough-active planting and fire management are required, along with long term monitoring and adaptive management.

This landscape is also facing a changing climate, and historic references may not be enough. We saw evidence of novel ecosystems, which may be suitable targets for future restoration efforts- the jury is still out on this one.

It was good to learn that agencies, consultants, industry, and individuals were taking a lead from SERI's publications and activities. We have a great responsibility-the future of many of these schemes is dependant on what we say and do. I am confident that SERI members are equal to this challenge- as they have been from the inception of the Society.

\author{
Jim Harris \\ Chair \\ Society for Ecological Restoration International
}

